\title{
Linx
}

Revue des linguistes de l'université Paris X Nanterre

11 | 1999

Typologie des langues, universaux linguistiques

\section{Le hongrois, langue SOV ou SVO : problèmes dans l'établissement de l'ordre de base}

Anna Sőrés

\section{(2) OpenEdition}

Journals

Édition électronique

URL : http://journals.openedition.org/linx/907

DOI : $10.4000 / \operatorname{lin} x .907$

ISSN : 2118-9692

Éditeur

Presses universitaires de Paris Nanterre

Édition imprimée

Date de publication : 1 décembre 1999

Pagination : 189-203

ISSN : 0246-8743

\section{Référence électronique}

Anna Sőrés, "Le hongrois, langue SOV ou SVO : problèmes dans l'établissement de l'ordre de base », Linx [En ligne], 11 | 1999, mis en ligne le 02 juillet 2012, consulté le 20 avril 2019. URL : http:// journals.openedition.org/linx/907 ; DOI : 10.4000/linx.907

Ce document a été généré automatiquement le 20 avril 2019

Département de Sciences du langage, Université Paris Ouest 


\title{
Le hongrois, langue SOV ou SVO: problèmes dans l'établissement de l'ordre de base
}

\author{
Anna Sőrés
}

\section{Introduction*}

1 Dans la plupart des langues (ou au moins dans celles sur lesquelles portent les analyses typologiques), l'établissement de l'ordre de base, ou, en un d'autres termes, de l'ordre dominant ne fait aucune difficulté. Ce paramètre est, en outre, l'un des rares qui permettent de procéder à une classification : il s'agira en effet de l'un des six types définis en fonction de la place respective du Sujet, du Verbe et de l'Objet, ou bien le classement peut se faire en langues OV/VO, ou encore, pour remonter jusqu'aux origines, on peut trouver la place d'une langue parmi les 24 types établis par Greenberg (1963). Dans l'annexe de cet article fondateur, parmi les langues appartenant au type SOV/Po/AN/GN, ilclasse le groupe "finno-ougrien à l'exception du groupe finnois", ce quisignifie, implicitement, que le hongrois devrait en faire partie. Le finnois ainsi que l'estonien, appartiennent au type SVO/Po/GN/AN.

2 Ailleurs, chez Mallinson et Blake (1981:132), le hongrois apparaît comme SVO ou libre, mais les auteurs évoquent le désaccord qui apparaît dans la littérature typologique où le hongrois est SVO selon Ruhlen, SOV selon Ultan et Greenberg (sans références exactes dans l'article). Notons encore qu'il est considéré comme SVO souple et relativement libre par Lazard (1990).

3 L'objectif de cet article est d'étudier dans le détail l'ordre des constituants fondamentaux, ensuite l'ordre Modifieur/Modifié (ou Régi/Régissant, p. ex. l'adjectif ou la relative seront considérés comme Modifieurs qui modifient le Nom), puis le rapport entre les deux.

4 La notion d'ordre de base est conçue de façon très différente par les linguistes. Depuis Greenberg (1963) qui ne l'a pas exactement défini, on a l'habitude de considérer l'ordre de 
base comme celui d'une phrase indépendante, assertive, stylistiquement non marquée, avec Sujet et Objet nominaux. Plus récemment, Dryer (1997, à propos de Hawkins 1983), propose de prendre en compte le nombre d'environnements dans lesquels la phrase se présente. Selon Dryer, ce type de phrase est loin d'être dominante parmi les énoncés.

Pour le hongrois, à l'heure actuelle nous ne connaissons pas d'étude sur la fréquence des types de phrases en synchronie ${ }^{1}$. Dans une étude diachronique, une telle analyse a été faite, nous y reviendrons plus tard. Par la suite, nous proposerons d'étudier l'ordre des constituants sur un corpus constitué pour ces objectifs.

\section{Observations sur l'ordre des constituants fondamentaux}

\subsection{Corpus}

6 L'hésitation constatée dans la littérature concernant l'ordre de base en hongrois s'explique avant tout par le manque d'informations sur l'ordre des mots dans les grammaires les plus courantes (à l'exception des plus récentes, d'approche générative). Mais même pour le locuteur natif, une certaine hésitation subsiste. Comme l'établissement de l'ordre de base n'est pas aussi simple qu'en anglais ou en français, il faut tester les possibilités. En effet, ce qui est le plus difficile à constater à propos d'une phrase hongroise, c'est de savoir si elle est «stylistiquement neutre ». Le problème se résout moins facilement à l'écrit qu'à l'oral, puisque l'accent d'intensité peut marquer, un objet focalisé, dans une phrase à ordre SOV qui, à l'écrit, paraîtrait « neutre » :

\begin{tabular}{|l|l|l|}
\hline Péter & levelet & ir. \\
\hline Pierre & lettre.COD & écrit. \\
\hline interprétation 1: Pierre écrit une lettre & \\
\hline interprétation 2 (à l'oral uniquement) : C'est une lettre que Pierre écrit (et pas ses devoirs) \\
\hline
\end{tabular}

7 Dans ce qui suit, nous allons étudier les phrases hongroises susceptibles de représenter l'ordre de base, et donc toutes les combinaisons possibles, à l'exception de phrases à verbe en tête, toujours emphatiques. Le corpus combine les lexèmes 'petit garçon', 'manger', 'gâteau'. Le sujet sera toujours exprimé par un SN défini.

8 Les variables sont les suivantes:

1. le degré de définitude du SN Objet, qui peut être défini avec article défini, indéfini avec article indéfini et indéfini avec article zéro².

2. l'aspect verbal: le verbe figurera d'abord au présent, ensuite au passé (forme unique), à l'aspect non-accompli, ensuite à l'accompli, sous forme de verbe préverbé.

9 Légende : Acc - accusatif, AACC - aspect accompli, ART.D - article défini, ART.I - article indéfini, $\mathrm{E}$ - présence d'emphase 


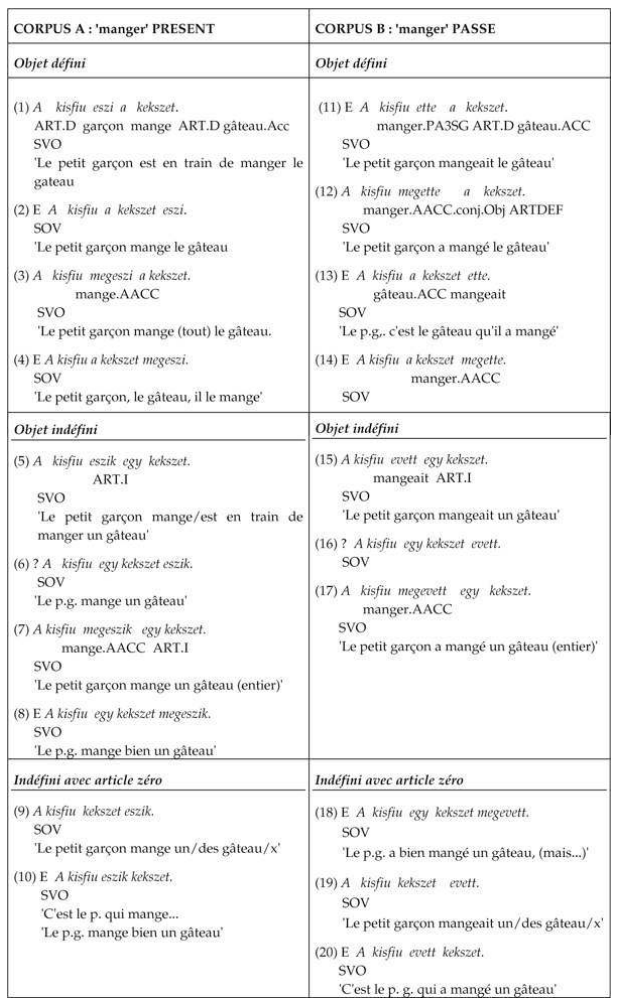

\subsection{Analyse}

10 La combinaison des lexèmes proposés permet la production de vingt phrases correctes dont neuf, précédées de ( $\mathrm{E}$ ) pour Emphase, ne peuvent pas être considérées comme stylistiquement non marquées. En effet, dans ces cas-là, le locuteur s'attend à une suite, $\mathrm{p}$. ex. (14) 'Le petit garçon, le gâteau, il l'a mangé, mais pas le déjeuner.' Dans le cadre proposé nous qualifions ces phrases simplement d'emphatiques, sans développer s'il s'agit de focalisation ou de thématisation.

11 Les phrases (6) et (16) sont précédées d'un point d'interrogation car leur interprétation pose problème. Quoique l'indéfini puisse être marqué par l'article indéfini ou par l'absence d'article, dans ces deux contextes-là le sens générique ne demande pas la présence de l'article indéfini (c'est ce qui pourrait être traduit par mange des gâteaux). En cas d'omission de l'article, (6) et (16) coïncideraient avec (9) et (19) respectivement. Le «? » signale donc le fait que l'usager hésite de les accepter, si egy est interprété comme article indéfini. En revanche, si l'on interprète egy comme numéral, il devrait porter l'accent d'intensité et la phrase, avec objet focalisé, serait emphatique, mais n'entrerait pas dans notre corpus qui est axé sur l'article.

Les phrases (9) et (19) permettent également une double interprétation : à l'écrit, elles peuvent passer pour «neutres ", mais à l'oral, avec accent d'intensité, elles peuvent être interprétées comme emphatiques, avec objet focalisé, alors que sans accent d'intensité elles peuvent être neutres. Notons encore que, dans ce dernier cas, le rapport OV sans article, donc le nom pris au sens générique, assure une plus grande cohésion entre le verbe et son objet (cf. un gros mangeur de gâteaux). Enfin, l'emploi du SN sans article est plus restreint, car il n'est compatible qu'avec l'aspect non-accompli, alors que le SN indéfini accepte les deux. 


\subsection{L'aspect verbal}

13 A propos des deux ordres SOV et SVO possibles, Dezsö (1982:243) affirme que SOV n'est pas possible avec des verbes perfectifs. En testant, dans les contextes précédents, les variations de la phrase A vadász lelötte a szarvast 'Le chasseur a tué (litt. tiré) le cerf', on constate qu'il y a en effet des différences par rapport aux possibilités qu'offre le verbe imperfectif 'manger', et ce dans trois contextes.

14 (Remarque : dans le corpus ci-dessous, la numérotation des exemples est continue, et le deuxième chiffre signale la phrase à laquelle l'exemple étudié correspond dans le corpus précédent)

\begin{tabular}{|l|l|l|l|l|l|}
\hline$(21)(1)$ & ${ }^{*} A$ & vadász & lövi & a & szarvast. \\
\hline & ART.D & chasseur & tirer.3SG & ART.D & cerf.ACC \\
\hline & SVO & & & & \\
\hline & 'Le chasseur tue le cerf' \\
\hline
\end{tabular}

\begin{tabular}{|l|l|l||l|l|l|}
\hline$(22)(2)$ & $*_{A}$ & vadász & a & szarvast & lövi. \\
\hline & Sov & & & & \\
\hline \hline$(23)(13)$ & $*_{A}$ & vadász & a & szarvast & lötte. \\
\hline & & & & & tirer.PASSE \\
\hline & SOV & & & & \\
\hline
\end{tabular}

On observe que l'incompatibilité ne se manifeste pas simplement entre perfectivité et SOV, car les phrases suivantes (avec V perfectif et SOV) sont correctes et neutres :

\begin{tabular}{|l|l|}
\hline$(24)(9)$ & A vadász szarvast lö (SOV) \\
\hline$(25)(19)$ & A vadász szarvast lött (SOV) \\
\hline
\end{tabular}

Il semblerait plutôt que le verbe perfectif n'accepte un objet défini qu'à l'aspect accompli, sous la forme préverbée du verbe, ce qui entraîne l'ordre SVO. C'est donc l'aspect accompli qui rend acceptables les phrases suivantes :

\begin{tabular}{|l|l|}
\hline$(26)(3)$ & A vadász lelövi a szarvast.(SVO) \\
\hline$(27)(12)$ & A vadász lelötte a szarvast.(SVO). \\
\hline
\end{tabular}


17 Pour certains locuteurs hungarophones la phrase (21) n'est pas complètement agrammaticale, mais plutôt focalisée, et qui demanderait un développement par une subordonnée. C'est d'ailleurs la raison qui amène F. Kiefer, le spécialiste de l'aspect en hongrois, à considérer l'aspect comme une catégorie de la sémantique de la phrase.

18 Si l'on accepte mes interprétations, on peut constater qu'avec ce verbe perfectif (lö 'tirer'), nous avons 8 ordres basiques sur 20 , la proportion est donc à peu près identique à celle obtenue avec le verbe manger.

\subsection{Bilan}

19 A partir des données du corpus, nous pouvons faire le relevé statistique suivant concernant les phrases «stylistiquement neutres». Si l'on omet les deux phrases problématiques, (6) et (16), on observe que sur dix-huit phrases, neuf sont neutres. Sur ces neuf phrases, sept sont SVO : (1) (3) (5) (7) (12) (15) (17) et deux SOV (9) (19).

L'analyse du corpus nous permet donc d'affirmer que l'ordre de base des constituants en hongrois est SVO. Cette observation fondée sur les données statistiques peut être corroborée par les corrélations entre les facteurs définitude, type de conjugaison, aspect verbal et ordre des mots, et ce selon la hiérarchie suivante :

Objet défini > conjugaison objective >accompli > VO

Objet indéfini > conjugaison subjective >accompli et non-accompli> VO

O. générique > conjugaison subjective $>$-accompli $>\mathrm{OV}$

21 Le caractère défini ou indéfini détermine le choix de la conjugaison, c'est une évidence en hongrois, mais l'introduction de deux autres traits, l'aspect accompli ${ }^{3}$ et l'ordre verbeobjet révèle une corrélation typologique qui permet d'expliquer la dominance de SVO par rapport à SOV :

1. SOV apparaît dans des conditions restreintes, par rapport à SVO, à savoir uniquement avec l'article zéro et à l'aspect non-accompli.

2. SVO est compatible avec les articles défini et indéfini, et avec les aspects accompli et non accompli, ce qui assure certainement une plus grande fréquence de cette construction.

3. En outre, les SN définis sont plus fréquents en discours que les indéfinis.

Avant de terminer cette partie de l'analyse, il nous semble important de revenir à l'aspect pragmatique qui n'a peut-être pas été suffisamment développé. Rappelons qu'en hongrois, la topicalisation ainsi que la focalisation (Sörés 1998) sont marquées uniquement par le changement de l'ordre des mots, sans aucune marque morphologique, avec, à l'oral, des moyens suprasegmentaux : l'accent d'intensité et la pause. Evidemment, la forme écrite, sur laquelle se fondent les études typologiques, ne permet aucune distinction. Donc, à la phrase française Paul aime Marie peuvent correspondre à l'oral trois énoncés hongrois, l'un qui pourrait être considéré comme neutre, un deuxième où le sujet serait topicalisé, Paul, il aime Marie et un troisième où le sujet serait focalisé, C'est Paul qui aime Marie. Or, la phrase hongroise écrite Pál szereti Marit neutralise ces différences. C'est ce qui rend difficile de qualifier une phrase comme neutre, surtout si ce critère se 
combine avec celui de la fréquence : on peut supposer qu'en discours ce sont les variantes non-neutres qui l'emportent.

\section{Ordre Modifieur/Modifié (ou Régissant/Régi)}

Sans entrer dans les détails concernant les théories qui portent sur ce sujet, nous pouvons étudier la réalisation d'un très grand nombre de traits. Il n'est évidemment pas possible d'atteindre à l'exhaustivité, pour plusieurs raisons. Entre autres, les paramètres qui figurent dans la littérature typologique ne sont pas tous interprétables dans toutes les langues. De plus, on va omettre les paramètres purement morphologiques, car leur étude (p. ex. celle de l'ordre des morphèmes grammaticaux) mériterait en soi un travail approfondi.

es traits qui seront étudiés ici sont empruntés en partie à Andersen (1983). On introduira également quelques traits portant sur la phrase complexe, qui ont été proposés par Dryer (1992) lors de la présentation de sa théorie de la direction du branchement.

Pour ce qui est de la présentation des données, elle reflète le problème théorique qui sera développé dans la partie suivante de notre travail. En effet, dans le cas du hongrois, il ne sera pas possible de s'appuyer sur l'hypothèse selon laquelle l'ordre respectif des modifiés et des modifieurs correspondrait à celui du verbe et de son objet. Le hongrois, quoique langue SVO, comme nous venons de le voir, n'est pas une langue « de type VO».

\subsection{Corpus}

Nous listerons dans le tableau ci-dessous les traits spécifiques de l'ordre OV, car ce sont ceux qui sont le plus souvent réalisés en hongrois ; dans les cas contraires, c'est-à-dire lorsque le hongrois présente l'ordre inverse, on note l'écart par un ${ }^{\circ}$.

\begin{tabular}{|l|l|}
\hline TRAITS SYNTAXIQUES & \\
\hline 1. N-POSTPOSITIONS & ebéd után 'après déjeuner' \\
\hline 2. COMPARAISON $:$ & $\begin{array}{l}\text { nagyobb mint Pál 'plus grand que Paul' } \\
\text { ADJ-Marqueur-Standard }\end{array}$ \\
\hline 3. ADJ+MARQUEUR de COMP & nagy+obb 'plus grand' \\
\hline 4. GROUPE ADPOS+V & $\begin{array}{l}\text { Moziba megyek. 'je vais au cinéma' } \\
\text { cinéma.LOC Verbe }\end{array}$ \\
\hline 5. PREDICAT+COPULE & tanár lesz 'il deviendra prof' \\
\hline 6. S-COMPLEMENTEUR & Tudom, hogy Pál beteg 'Je sais que Paul est malade' \\
\hline 7. S-ADV.SUBORD & mert Pál jön'parce que Paul viendra' \\
\hline 8. N-RELATIVE & a levél, amit olvasok 'la lettre que je lis' \\
\hline
\end{tabular}




\begin{tabular}{|c|c|}
\hline MODIFIEURS NOMINAUX & \\
\hline 9. ADJECTIF-N & szép kert 'beau jardin' \\
\hline 10. GENITIF-N & Péternek a kertje 'le jardin de Pierre' \\
\hline 11. DETERMINANT-N & (ez) a kert 'ce jardin' \\
\hline 12. NUMERAL-N & két gyerek 'deux enfants' \\
\hline 13. ADV-ADJ & rendkivül nagy 'extrêmement grand' \\
\hline MODIFIEURS VERBAUX & \\
\hline 14. ADVERBE-V & gyorsan fut 'il court vite' \\
\hline 15. V-AUXILIAIRE & olvasni fog 'il va lire' \\
\hline 16. V-INTENTION & menni akar 'il veut partir' \\
\hline 17. V+POTENTIEL & olvas+hat 'il peut lire' \\
\hline 18. $\mathrm{V}+\mathrm{CAUSATIF}$ & olvas+tat 'il fait lire' \\
\hline 19. V+REFLECHI & mos+akodik 'se lave' \\
\hline AUTRES TRAITS & \\
\hline 20. V-INTERROGATIVE & Van-e ?'Y en a-t-il? \\
\hline 21. NOM-TITRE & Kiss doktor 'docteur K.' \\
\hline 22. N.de FAMILLE+Prén. & Kiss Pál 'Paul K.' \\
\hline
\end{tabular}

\subsection{Analyse}

Sur les vingt-deux traits étudiés, le hongrois en possède dix-huit qui caractérisent le type OV et quatre qui correspondent au comportement VO. Il faut tout de suite souligner que chacun des quatre traits VO se manifeste au niveau de la phrase, alors qu' au sein des syntagmes les modifieurs nominaux et verbaux définissent clairement une langue OV. Notons qu'ici, nous considérons la position de la relative comme un trait syntaxique, même s'il s'agit d'un «modifieur du N ».

28 On peut ajouter deux autres traits qui, de façon implicite, ont déjà été étudiés dans ce qui précède. Dryer (1997) propose de dissocier, dans SVO, le rapport fondamental entre le Verbe et son Objet d'une part, et l'ordre respectif du Sujet et du Verbe d'autre part. Les deux traits complémentaires seraient ainsi SV et VO.

En hongrois, la place du Sujet est majoritairement préverbale (SV), VS n'apparaissant que dans les incises et en cas de focalisation, d'interrogation, etc. Pour ce qui est de la place 
de l'objet par rapport au verbe, nous venons d'observer que l'ordre de base est SVO ; on a donc la séquence VO.

Il s'agit ainsi au total de vingt-quatre traits. Dans 6 cas sur 24, le comportement du hongrois est celui d'une langue VO, et ce toujours au niveau de la phrase, simple ou complexe. Ces six traits sont:

\begin{tabular}{|l|l|l|l|l||l|}
\hline Vo & SV & NRel & Adj-M-St & COMP-S & AdvSubo-S. \\
\hline
\end{tabular}

31 Notons cependant que la décision de Dryer de distinguer les complémenteurs et les adverbes de subordination ne fait pas l'unanimité.

\subsection{Remarques complémentaires sur les " écarts "}

Avant d'aborder la réflexion sur les aspects théoriques, nous pouvons faire quelques remarques sur deux traits précis, à savoir la comparaison et la relative. Sur les autres points, une étude approfondie reste à faire.

La comparaison de l'adjectif peut se faire en effet selon les deux ordres. L'ordre qui correspondrait à OV est le suivant :

\begin{tabular}{|l|l|l|}
\hline Pálnál & nagyobb & vagyok. \\
\hline \hline Paul-LOC & grand-MARQ & suis \\
\hline ST & Adj+M & Verbe \\
\hline \multicolumn{2}{|l|}{ 'Je suis plus grand que Paul' } \\
\hline
\end{tabular}

Dans cette phrase, le «standard» (Paul) est focalisé ; l'ordre neutre, beaucoup plus courant, correspond à Vo (cf. tableau) :

\begin{tabular}{|l|l|l|}
\hline Nagyobb & vagyok & mint Pál. \\
\hline grand-M & suis & que Paul \\
\hline Adj+M & Verbe & ST \\
\hline
\end{tabular}

On est donc amené à considérer l'ordre du type VO comme stylistiquement neutre. Par ailleurs, la place de la subordonnée relative par rapport au nom n'a apparemment pas subi de changement au cours de l'histoire attestée de la langue, c'est-à-dire depuis le XI ${ }^{\mathrm{e}}$ siècle. L'étude qui pourrait être menée devrait porter d'abord sur la relation (voire la corrélation) de la place de l'adjectif et du nom, comparée à celle de la relative. Ensuite, il reste à étudier la place des relatives non-phrastiques. En effet, le hongrois, comme l'allemand, utilise assez souvent des relatives participiales qui, comme les adjectifs, précèdent le nom : 


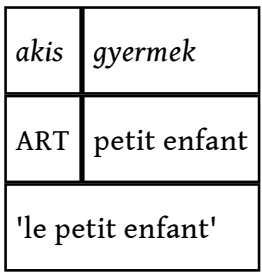

\begin{tabular}{|l|l|l|}
\hline az & alvó & gyermek \\
\hline ART & dormant & enfant \\
\hline
\end{tabular}

\begin{tabular}{|l|l|l|l|}
\hline$a$ & gyermek, & aki & alszik \\
\hline ART & enfant & REL & dort \\
\hline \hline 'l'enfant qui dort'
\end{tabular}

Dans le cas de la relative il existe donc également deux ordres de constructions possibles : postposée au $\mathrm{N}$ quand elle est verbale, antéposée quand elle est participiale.

\subsection{Rappel des observations}

L'analyse que nous avons faite sur l'ordre des constituants et sur celui des Modifieurs/ Modifiés nous a permis de faire les observations suivantes:

- nous pouvons affirmer sans hésitation que l'ordre de base des constituants de niveau phrastique est SVO ;

- en hongrois, l'ordre des constituants fondamentaux ne permet pas de prévoir l'ordre respectif des autres modifieurs : langue SVO mais pas VO, si par VO on entend, par extension, le fait que les modifieurs suivent le modifié comme l'objet suit le verbe ;

- l'ordre Modifieur/Modifié au sein des syntagmes est majoritairement celui du type dit OV, alors qu'au niveau de la phrase, soit simple, soit complexe, l'ordre des items correspond au comportement d'une langue VO.

Pour terminer, nous essayerons d'analyser cette « disharmonie » avant tout du point de vue diachronique.

\section{L'évolution du hongrois}

Le proto-ouralien est reconstruit comme langue SOV suivant l'ordre Modifieur/Modifié. Beaucoup de langues finno-ougriennes sont SOV, mais celles que nous connaissons le mieux, à savoir le finnois, l'estonien et le hongrois sont SVO. Il s'est déroulé comme pour l'indo-européen, un changement typologique de SOV vers SVO.

Pour le hongrois, des études diachroniques précises ont été menées (Wacha 1995). Le changement de SOV en SVO s'amorce déjà en proto-hongrois (jusqu'au $\mathrm{X}^{\mathrm{e}}$ siècle), accompagné du développement du marquage systématique de l'objet. Selon les statistiques, OV et VO sont attestés pendant les siècles qui suivent (ancien hongrois 
précoce, jusqu'au milieu du XIVe), et dans Vo l'objet est très souvent défini. Pour la période de l'ancien hongrois tardif (1350-1550) il existe une étude statistique qui montre déjà une nette supériorité de SVO : 44,3\% par rapport à SOV 21,6\%.

41 Les mêmes études démontrent en revanche la stabilité incontestable d'autres traits sériels, en particulier la place de l'adjectif (AN) et celle du génitif (GN) (ainsi que Négation +élément nié, Prédicat+Copule, traits que nous ne développons pas ici) qui sont attestés depuis le proto-hongrois.

Ces divers remarques concernant l'évolution du hongrois révèlent un phénomène capital : il semblerait qu'en diachronie comme en synchronie, l'ordre des mots dans le Syntagme Nominal est rigide alors que l'ordre des constituants montre une grande variétét.

\section{Dans quel type classer le hongrois?}

Tout en considérant d'une part la classification comme une étape possible mais non nécessaire de la typologie, et d'autre part la possibilité que l'ordre des mots offre à une classification, nous sommes obligé d'admettre que la place du hongrois n'est pas facile à trouver dans les approches proposées.

Greenberg suggère de le classer dans le type SOV/Po/AN/GN, mais nous venons de voir qu'il appartient au type SVO/Po/AN/GN.

Il n'est pas possible de le classer selon OV ou VO non plus. En effet, la séquence OV correspond à (S)OV, à OV(S) et à $\mathrm{O}(\mathrm{S}) \mathrm{V}$, de même, VO correspond à (S)VO, à VO(S) et à $\mathrm{V}$ (S)O, si l'on fait abstraction de la place du sujet. Dans cette perspective, le hongrois, langue SVO selon l'ordre des constituants, devrait être VO, alors que, pour la plupart des traits étudiés elle montre les caractéristiques d'une langue OV.

L'approche de Dryer (1997) qui propose de dissocier SV et OV pourrait permettre de conférer au hongrois un statut de langue à verbe-final, mais seulement si, de façon paradoxale, on ne considère OV que symboliquement, c'est-à-dire comme valable pour tout rapport Modifieur/Modifié, et dans ce cas seul ferait exception l'ordre du V et de son objet. Evidemment, ce serait purement spéculatif, puisque l'auteur ne semble pas avoir conçu la séquence OV de telle façon.

Il serait en tout cas intéressant de voir si le cas du hongrois est isolé ou bien s'il existe d'autres langues dans lesquelles l'ordre de base des constituants fondamentaux ne détermine pas l'ordre des modifieurs. On peut penser que l'aspect diachronique ne peut être négligé. En effet, les langues SVO et en particulier les langues romanes ne sont pas des langues VO harmoniques, et ce pour des raisons diachroniques. Selon la conception de Greenberg sur le dynamisme (1963) toute déviation par rapport à l'ordre dominant s'explique par le fait que les langues sont constamment en train de changer. Néanmoins, les traits OV du hongrois actuel ne montrent aucun signe d'un changement éventuel.

\section{Conclusions, perspectives}

Ce qui vient d'être dit nous permet de faire quelques réflexions sur la chronologie des changements. Il semblerait qu'en hongrois, l'ordre SOV, primitif, soit maintenu avec l'article zéro et que les autres paramètres sériels (que nous avons appelé «ordre 
Modifieur/Modifié ») répondent également à la tendance OV. Le premier indice d'un changement en VO se manifeste au niveau des constituants fondamentaux ce qui parait un processus contraire à ce qui s'est déroulé lors de l'évolution du latin jusqu'aux langues romanes (Marchello-Nizia 1995 :111-112). La réflexion doit donc être poursuivie dans la direction que désignent Lehmann et Ratanajoti (1975 : 158-9, cité par Andersen op.cit. :25) qui suggèrent qu'au cours d'un changement de OV vers Vo les modifieurs verbaux et les compléments sont affectés les premiers (comme cela semble être le cas dans les dialectes indo-européens). La chronologie des changements reste donc une piste à développer.

Pour ce qui est des corrélations, il est reconnu que l'ordre de certaines paires d'éléments grammaticaux est en harmonie avec l'ordre respectif du Verbe et de l'objet. A la suite des remarques de Greenberg, Dryer a consacré une très vaste étude à ces paires correlationnelles et à l'explication de leur existence. Selon lui, les traits AN/ DemN/ AdvAdj/ NegV/ Temps/Asp ne sont pas correlationnels. Nous n'avons pas pris en considération les deux derniers traits. Pour les trois premiers, nous pouvons dire qu' en hongrois, on a AN/ DemN/ AdvAdj, conformément à OV. Mais que dire de ces corrélations, dès lors que, comme on l'a vu, le comportement VO qui correspondrait à l'ordre de base en hongrois n'apparaît que dans 6 traits sur 24 ? Les discussions sur les corrélations ou sur Modifieurs et Modifiés portent sur des problèmes concrets, alors qu'ici la difficulté semble plus théorique : peut-on parler de corrélation des traits sériels avec l'ordre respectif du $\mathrm{V}$ et de l'O, s'il y a une nette contradiction entre la présence de VO dans l'ordre de base et la présence de OV dans la majorité des autres traits ? Ou bien le décalage entre l'ordre des constituants et celui de modifieurs est-il un simple fait diachronique, comme le suggère Greenberg ? Est-ce que ce fait ne s'explique pas plutôt en synchronie?

Etant donné que les écarts par rapport à OV se présentent au niveau de la Phrase, soit simple, soit complexe, on peut se demander si on peut mettre en dénominateur commun l'ordre des mots au sein d'un SN ou d'un SV et l'ordre de ces syntagmes qui constituent les phrases, ou encore l'ordre de la phrase matrice et de l'enchassée.

51 L'exemple du hongrois suggérerait qu'il y a ici des problèmes à reconsidérer, surtout s'il s'avérait qu'il existe d'autres langues ayant le même comportement. Il n'est donc pas possible de répondre à ces questions avant d'avoir travaillé sur un plus grand échantillon de langues.

\section{BIBLIOGRAPHIE}

ANDERSEN, Paul Kent (1983) Word Order Typology and Comparative Constructions, John Benjamins, Amsterdam/Philadelphia

DEzsö, Lászlo (1982) Studies in Syntactic Typology and Contrastive Grammar, Mouton, The HagueParis-New York

DRYER, M. S. (1992) « The Greenbergian Word Order Correlations », in Language 68/1 : 81-138

---- (1997) « On the six-way word order typology », in Studies in Language, 21 :1, 69-103. 
GREENBERG, J.H. $\left(1963,1966^{2}\right)$ « Some Universals of Grammar with Particular Reference to the Order of Meaningful Elements », in : Greenberg éd. Universals of Language, Cambridge, The MIT Press, 73-113.

HAWKINS, J. A. (1983) Word Order Universals, Academic Press Inc. San Diego, California

LAZARD, Gilbert (1990) Caractéristiques actantielles de l'« européen moyen type » in Toward a Typology of European Languages, éd. Bechert, J. et al. Mouton de Gruyter, Berlin-New York

Mallinson, G. et BlaKe, B.J. (1981) Language Typology. Crosslinguistic studies in Syntax; North Holland Publishing Co. Amsterdam

MARCHELlo-NizIA, Christiane (1995) L'évolution du français. Ordre des mots, démonstratifs, accent tonique, Armand Colin, Paris

SÖRES, Anna (1998) «Topic, focus et ordre des mots en hongrois », in La Thématisation dans les langues, éd. Guimier, C. Peter Lang, Berne : 217-230.

STEEL, Susan (1987) « Word order variations : a typological study »; in : Universals of human language I-IV. éd. Greenberg, J. H. Stanford, Stanford University Press.

WACHA, Balázs (1995) «A mondat szorendje » (L'ordre des mots dans la phrase) in A magyar nyelv történeti nyelvtana (Grammaire historique de la langue hongroise) , éd. Benkö L. Akadémiai Kiado, Budapest

\section{NOTES}

*. Je tiens à remercier D. Fattier, D. Leeman et C. Marchello-Nizia de leurs suggestions et de leurs conseils.

1. Il n'existe pas d'étude précise, mais une brève observation de quelques textes littéraires modernes que j'ai faite semble confirmer une fréquence peu élevée de phrases neutres, avec sujet nominal surtout.

2. La conjugaison du Verbe transitif possède un double paradigme suivant le caractère défini ou indéfini de l'objet, appelé respectivement conjugaison objective et subjective ;

3. Faute d'avoir fait des recherches précises sur d'autres langues, nous ne pouvons citer que quelques exemples pris dans la littérature sur des données comparables concernant la définitude et l'aspect. Selon Li et Thompson (1975:1, cités par Steele $1978: 596)$, en chinois l'ordre OV s'emploie avec objet défini, alors que VO s'emploie avec objet indéfini, ce qui est le contraire de ce que l'on trouve en hongrois, et en même temps, de ce qu'indique Dryer (1997) pour le papago. L'importance de l'aspect verbal dans l'ordre de $\mathrm{V}$ et de $\mathrm{O}$ est illustré par Mallinson et Blake (op.cit.161) sur le lendu, où l'ordre SVO (sans article) marque l'aspect perfectif, SOV l'imperfectif, sans aucun autre changement que celui de l'ordre des termes. Afin de confirmer l'importance de ces facteurs dans l'ordre des constituants, on aurait besoin de multiplier les données exactes.

4. Selon Dezsö (1982:181, 243), l'une des raisons du changement remonte probablement à l'apparition de l'aspect accompli, ce qui a été accompagné de la naissance de l'article défini. Il semble que le changement de l'ordre des mots accompagnant la naissance de l'aspect accompli peut être comparée à ce qui se passe dans les langues slaves, sans toutefois arriver jusqu'à l'article. 


\section{AUTEUR}

\section{ANNA SŐRÉS}

Université Paris X Nanterre - EA 372 